\title{
SELLING A LARGE STOCK POSITION: A STOCHASTIC CONTROL APPROACH WITH STATE CONSTRAINTS*
}

\author{
M. PEMY ${ }^{\dagger}$, Q. ZHANG ${ }^{\ddagger}$, AND G. YIN ${ }^{\S}$
}

\begin{abstract}
A common practice for stock-selling decision making is often concerned with liquidation of the security in a short duration. This is feasible when a relative smaller number of shares of a stock is treated. Selling a large position during a short period of time in the market frequently depresses the market, resulting in poor filling prices. In this work, liquidation strategies are considered for selling much smaller number of shares over a longer period of time. By using a fluid model in which the number of shares are treated as fluid, and the corresponding liquidation is dictated by the rate of selling over time. Our objective is to maximize the expected overall return. The problem is formulated as a stochastic control problem with state constraints. Using the method of constrained viscosity solutions, we characterize the dynamics governing the value function and the associated boundary conditions. Numerical algorithms are also provided along with an illustrative example for demonstration purposes.
\end{abstract}

Keywords: Optimal control, state constraint, selling rule.

1. Introduction. In a financial market, decision making in stock liquidation is crucial for trading and portfolio management. In his book [9], Øksendal formulated the optimal selling decision making as an optimal stopping problem and provided a closed-form solution. By mean of a smooth fit technique, the underlying problem may be treated as a free boundary value problem, which may be traced back to McKean [8] in the 1960's. These results are extended to incorporate possible regime switching by Guo and Zhang [5] with a two-state Markov chain. In [5], using a smooth-fit technique, the optimal stopping problem was converted to a set of algebraic equations under certain smoothness conditions. Closed-form solutions were obtained in these cases.

Alternatively, one may consider a selling rule determined by two threshold levels, a target price and a stop-loss limit. A selling decision is made whenever the price reaches either the target price or the stop-loss limit. The objective is to choose these threshold levels to maximize an expected return. In [16], the optimal threshold levels were obtained by solving a set of two-point boundary value problems for a model with regime switching. If the Markov chain that governs the regime switching has only two

\footnotetext{
*This research was supported in part by the National Science Foundation.

†Department of Mathematics, Towson University, Towson, MD 21252-0001, E-mail: mpemy@towson.edu

${ }^{\ddagger}$ Department of Mathematics, Boyd GSRC, The University of Georgia, Athens, GA 30602-7403, E-mail: qingz@math.uga.edu

$\S$ Department of Mathematics, Wayne State University, Detroit, MI 48202, E-mail: gyin@math.wayne.edu
} 
states, the corresponding two-point boundary value problem has an analytic solution and the optimal solution can be obtained in a closed form. When the modulating Markov chain has a large number of states, a two-time-scale approach is suggested by Zhang, Yin, and Liu [17] for obtaining near-optimal selling strategies. From a different angle, Yin, Liu, and Zhang [14], developed a class of recursive algorithms based on stochastic approximation methods for stock liquidation problems. The idea is to turn the optimal stopping problem to a parametric stochastic optimization problem by focusing on threshold-type strategies. Along with convergence and rates of convergence of the algorithms, simulation examples were presented and the computation results were compared to the analytic solutions when the Markov chain having two states. Markov chains have more than two states as well as real data are dealt with. We point out that the method can be extended to treat the case that the precise model is not available; see [15] for demonstrations using NASDAQ market data. Recently, Helmes [6] considered computational issues of the selling rule using a linear programming approach. Pemy and Zhang [11] studied a selling rule in which the liquidation is constrained to be within a pre-specified time period.

A feature shared by the aforementioned papers is that the selling has to be done all at once. This is feasible when a relative smaller number of shares of a stock is considered. Selling a large shares of a stock in a market frequently depresses the market if it is sold in a short period of time resulting in poor filling prices. Typically, a strategy for selling stock of large size is to sell much smaller portion of the stock over a longer period of time. This paper is devoted to the problem of selling a large block of stock. We treat the selling rule problem using a fluid model in the sense that the liquidation is dictated by the rate of selling over time. The objective is to maximize the overall return. The underlying control problem has state constraints. This makes the problem much more difficult to analyze. Using constrained viscosity solution techniques (see Soner [13]) we characterize the dynamics governing the value function and to treat boundary conditions. Instead of treating the stock price alone, we consider a pair of variables, namely, the stock price as well as the size of the stock at time $t$. An easily implementable optimal selling strategy is obtained, which presents a threshold-like selling rule. Roughly, as demonstrated in the numerical experiments, the state-dependent "threshold" curve separates the entire region into two parts, selling region and no selling region. In addition, we consider a finite difference method for solving the associated Hamilton-Jacobi-Bellman (HJB) equations and demonstrate its convergence.

The rest of the paper is organized as follows. In the next section, we formulate the problem as a constrained stochastic control problem and provide needed assumptions. Section 3 studies properties of the value (reward) function and establishes that it is 
the only viscosity solution to an HJB equation. Also provided are the associated boundary conditions and optimal selling rate. In Section 4, we consider a finite difference method for solving the associated HJB equations and derive its convergence. In Section 5, we present a numerical example demonstrate how to apply the method to find the optimal selling rule. Section 6 concludes the paper. To preserve the flow of presentation, proofs of technical results are all relegated to an appendix.

2. Problem Formulation. Given a stock price $X(t)$ that follows the dynamics

$$
d X(t)=X(t)(\mu d t+\sigma d W(t)), \quad X(0)=x,
$$

where $\mu$ and $\sigma$ are constants representing the stock return rate and volatility and $W(\cdot)$ is a standard Brownian motion. Let $Y(t)$ be the number of shares of a stock sold by time $t$. We have

$$
d Y(t)=u(t) d t, \quad Y(0)=y .
$$

Let $S=(0, \infty) \times[0, N]$ be the state space where $N<\infty$ is the total number of the stock to be sold. Let $u(t)$ denote the selling rate which is the control variable. Let $\Gamma=[0,1]$ denote the control set.

Definition 2.1. We say that a control $u(\cdot)$ is admissible with respect to the initial values $(x, y) \in(0, \infty) \times[0, N]$, if, (i) $u(\cdot)$ is an $\mathcal{F}_{t}=\sigma\{X(s): s \leq t\}$ adapted; (ii) $u(t) \in \Gamma$ for all $t \geq 0$; (iii) the corresponding state process $(X(t), Y(t)) \in S$ for all $t \geq 0$. We use $\mathcal{A}=\mathcal{A}(x, y)$ to denote the set of all admissible controls.

Let $\rho>0$ be the discount rate. We assume $\rho>\mu$, and define

$$
J(x, y, u(\cdot))=E \int_{0}^{\infty} e^{-\rho t} X(t) u(t) d t .
$$

The problem is to choose the rate of selling $u(\cdot) \in \mathcal{A}$ so as to maximize $J(x, y, u(\cdot))$. The value function is

$$
v(x, y)=\sup _{u(\cdot) \in \mathcal{A}} J(x, y, u(\cdot)) .
$$

Formally, the HJB equation is given as follows:

(4) $\rho v(x, y)=\max _{u \in \Gamma}\left[\mu x \frac{\partial v(x, y)}{\partial x}+u \frac{\partial v(x, y)}{\partial y}+\frac{1}{2} \sigma^{2} x^{2} \frac{\partial^{2} v(x, y)}{\partial x^{2}}+x u\right], 0 \leq y \leq N$.

DEFINITION 2.2. $v(x, y)$ is a constrained viscosity solution of (4), if,

(1) $v(x, y)$ is a viscosity supersolution of $(4)$ on $(0, \infty) \times[0, N]$, i.e.,

$$
\rho v\left(x_{0}, y_{0}\right)-\max _{u \in \Gamma}\left[\mu x_{0} \frac{\partial \phi\left(x_{0}, y_{0}\right)}{\partial x}+u \frac{\partial \phi\left(x_{0}, y_{0}\right)}{\partial y}+\frac{1}{2} \sigma^{2} x_{0}^{2} \frac{\partial^{2} \phi\left(x_{0}, y_{0}\right)}{\partial x^{2}}+x_{0} u\right] \geq 0,
$$

whenever $\phi(x, y) \in C^{2}$ such that $v(x, y)-\phi(x, y)$ has a local minimum at $\left(x_{0}, y_{0}\right) \in(0, \infty) \times[0, N] ;$ 
(2) $v(x, y)$ is a viscosity subsolution of $(4)$ on $(0, \infty) \times(0, N)$, i.e.,

$$
\rho v\left(x_{0}, y_{0}\right)-\max _{u \in \Gamma}\left[\mu x_{0} \frac{\partial \psi\left(x_{0}, y_{0}\right)}{\partial x}+u \frac{\partial \psi\left(x_{0}, y_{0}\right)}{\partial y}+\frac{1}{2} \sigma^{2} x_{0}^{2} \frac{\partial^{2} \psi\left(x_{0}, y_{0}\right)}{\partial x^{2}}+x_{0} u\right] \leq 0,
$$

whenever $\phi(x, y) \in C^{2}$ such that $v(x, y)-\phi(x, y)$ has a local maximum at $\left(x_{0}, y_{0}\right) \in(0, \infty) \times(0, N)$.

3. Properties of Value Function. This section presents properties of the value function. We first show that the value function is Lipschitz continuous. Then we show that the value function is in fact the unique solution of the HJB equation in the sense of viscosity solution. Next, boundary conditions are provided. Finally, the optimal selling rules is specified.

\subsection{Properties of Value Function.}

Lemma 3.1. The following assertions hold.

(a) For each $x, v(x, y)$ is nonincreasing in $y$.

(b) $v(x, y)$ is Lipschitz continuous in $(x, y)$. More precisely,

$$
\left|v\left(x_{1}, y_{1}\right)-v\left(x_{2}, y_{2}\right)\right| \leq \frac{\left|x_{1}-x_{2}\right|}{\rho-\mu}+\left(x_{2}+1\right)\left|y_{1}-y_{2}\right|,
$$

for $\left(x_{1}, y_{1}\right)$ and $\left(x_{2}, y_{2}\right)$ in $(0, \infty) \times[0, N]$.

TheOREM 3.2. The value function $v(x, y)$ is the unique constrained viscosity solution on $(0, \infty) \times[0, N]$.

3.2. Boundary Condition. Let us consider the case $v \in C^{2}$. In this case, $v(x, y)$ satisfies the HJB equation (4). Take $\phi(x, y)=v(x, y)+\beta(y-N)$ with $\beta>$ 0 . Then, $v(x, y)-\phi(x, y)$ reaches minimum at $\left(x_{0}, N\right)$. In view of the viscosity supersolution, this implies

$$
\max _{u \in \Gamma}\left\{u \frac{\partial \phi\left(x_{0}, N\right)}{\partial y}+x u\right\}=\max _{u \in \Gamma}\left\{u\left(\frac{\partial v\left(x_{0}, N\right)}{\partial y}+\beta\right)+x u\right\} \leq \max _{u \in \Gamma}\left\{u \frac{\partial v\left(x_{0}, N\right)}{\partial y}+x u\right\},
$$

which leads to the boundary condition

$$
\frac{\partial v\left(x_{0}, N\right)}{\partial y}+x_{0} \leq 0
$$

There is no constraint at $y=0$ because $u(t) \geq 0$ which makes $Y(t) \geq 0$ for all $t$.

3.3. Optimal Selling Strategy. In view of the standard verification theorem given in Fleming and Rishel [4] (See Zhou [18] for the corresponding version under the viscosity solution framework), the optimal selling rate should have the following form:

$$
u^{*}(x, y)= \begin{cases}0, & \text { if } \frac{\partial v(x, y)}{\partial y}+x<0 \\ 1, & \text { if } \frac{\partial v(x, y)}{\partial y}+x \geq 0\end{cases}
$$


This optimal selling strategy is simple and easily implemented. It offers much insight for the trading practice. As shown in (5), the optimal selling strategy is to sell one unit of stock (the unit here could be 1,000 shares, for example) whenever $\frac{\partial v(x, y)}{\partial y}+x \geq 0$, otherwise no sell action will be taken. Intuitively, also as shown in the numerical example to follow, for a given $y$, the optimal selling rule should be determined by a threshold level $x^{*}$ so that one sells at full rate if $x>x^{*}$ (when the price is attractive) and sells nothing if $x<x^{*}$. Similarly, for a given $x$, the optimal selling rule should be: There exists a $y^{*}$ such that one sells at full rate if $y<y^{*}$ (when more shares yet to be sold) and sells nothing when $y>y^{*}$. The simplicity of the selling rule should be particularly welcomed by the practitioners in financial market.

4. A Numerical Scheme and Its Convergence. In this section, we consider an explicit finite difference scheme and show that it converges to the unique viscosity solution of equation (4). Given a positive integer $M$, we consider the following optimal control problem with value function $v_{M}$

$$
v_{M}(x, y)=\sup _{u \in \mathcal{A}(x, y)} E \int_{0}^{\infty} e^{-\rho t} \min (X(t), M) u(t) d t .
$$

The corresponding HJB equation is given by

$$
\begin{gathered}
\rho v_{M}(x, y)=\max _{u \in \Gamma}\left[(\mu x) \frac{\partial v_{M}(x, y)}{\partial x}+u \frac{\partial v_{M}(x, y)}{\partial y}+\frac{1}{2} \sigma^{2} x^{2} \frac{\partial^{2} v_{M}(x, y)}{\partial x^{2}}\right. \\
+\min (x, M) u], 0 \leq y \leq N
\end{gathered}
$$

The corresponding Hamiltonian is

$$
\begin{aligned}
\mathcal{H}_{M}\left(x, y, v, \mathrm{D}_{x} v, \mathrm{D}_{y} v, \mathrm{D}_{x}^{2} v\right)= & \max _{u \in \Gamma}\left[\mu x D_{x} v(x, y)+u D_{y} v(x, y)\right. \\
& \left.+\frac{1}{2} \sigma^{2} x^{2} D_{x x} v(x, y)+\min (x, M) u\right]-\rho v(x, y) .
\end{aligned}
$$

As in the previous section, we can show that the value function $v_{M}$ is the unique constrained viscosity solution of the equation

$$
\mathcal{H}_{M}\left(x, y, v, \mathrm{D}_{x} v, \mathrm{D}_{y} v, \mathrm{D}_{x}^{2} v\right)=0, \text { for }(x, y) \in S .
$$

Moreover, $v_{M} \rightarrow v$ as $M \rightarrow \infty$, for all $(x, y)$. Thus, we need only find the numerical solution for $v_{M}$. We introduce the following change of variable. For each $x>0$, define $z=\log x$ and we set $v(x, y)=w(\log x, y)$. Then

$$
\begin{gathered}
\frac{\partial v}{\partial x}=\frac{\partial w}{\partial z} \frac{1}{e^{z}}, \text { and } \frac{\partial^{2} v}{\partial x^{2}}=\frac{\partial^{2} w}{\partial z^{2}} \frac{1}{e^{2 z}}-\frac{1}{e^{2 z}} \frac{\partial w}{\partial z}, \\
\frac{\partial v}{\partial y}=\frac{\partial w}{\partial y} .
\end{gathered}
$$


Therefore, the Hamiltonian defined in equation (7) becomes

$$
\begin{aligned}
\mathcal{H}_{M}\left(z, y, v, \mathrm{D}_{z} w, \mathrm{D}_{y} w, \mathrm{D}_{z}^{2} w\right)=\max _{u \in \Gamma}\left[\left(\mu-\frac{1}{2} \sigma^{2}\right) D_{z} w(z, y)+u D_{y} w(z, y)\right. \\
\left.+\frac{1}{2} \sigma^{2} D_{z z} w(x, y)+\min \left(e^{z}, M\right) u\right]-\rho w(z, y) .
\end{aligned}
$$

We will approximate the solution of the equation

$$
\begin{gathered}
\max _{u \in \Gamma}\left[\left(\mu-\frac{1}{2} \sigma^{2}\right) \frac{\partial w(z, y)}{\partial z}+u \frac{\partial w(z, y)}{\partial y}+\frac{1}{2} \sigma^{2} \frac{\partial^{2} w(z, y)}{\partial z^{2}}+\min \left(e^{z}, M\right) u\right] \\
-\rho w(z, y)=0,
\end{gathered}
$$

for $(z, y) \in \mathbb{R} \times[0, N]$. It can be shown that equation (10) has a unique viscosity solution denoted by $w_{M}$. Let $B(\mathbb{R} \times[0, N])$ be the space of bounded functions $u(z, y)$ defined on $\mathbb{R} \times[0, N]$ and continuous in $(z, y)$. Let $h$ with $0<h<1$ be the stepsize for variable $z$ and $k$ with $0<k<1$ be the stepsize for $y$. We consider the finite difference operators $\Delta_{z}, \Delta_{y}$ and $\Delta_{z}^{2}$ defined by

$$
\begin{aligned}
& \Delta_{x} w(z, y)=\frac{w(z+h, y)-v(z, y)}{h} \\
& \Delta_{y} w(z, y)=\frac{w(z, y+k)-w(z, y)}{k}
\end{aligned}
$$

and

$$
\Delta_{z}^{2} w(z, y)=\frac{w(z+h, y)+w(z-h, y)-2 w(z, y)}{h^{2}} .
$$

The corresponding discrete version of equation (10) is given by

$$
\begin{aligned}
\rho w(z, y)= & \max _{u \in \Gamma}\left[\left(\mu-\frac{1}{2} \sigma^{2}\right) \frac{w(z+h, y)-w(z, y)}{h}+u \frac{w(z, y+k)-w(z, y)}{k}\right. \\
& \left.+\frac{1}{2} \sigma^{2} \frac{w(z+h, y)+w(z-h, y)-2 w(z, y)}{h^{2}}+\min \left(e^{z}, M\right) u\right] .
\end{aligned}
$$

Rearranging terms, we obtain

$$
\begin{gathered}
\max _{u \in \Gamma}\left[\frac { 1 } { \rho + ( \mu - \frac { \sigma ^ { 2 } } { 2 } ) \frac { 1 } { h } + \frac { \sigma ^ { 2 } } { h ^ { 2 } } + \frac { u } { k } } \left(w(z, y+k) \frac{u}{k}+w(z+h, y)\left(\left(\mu-\frac{\sigma^{2}}{2}\right) \frac{1}{h}+\frac{\sigma^{2}}{2 h^{2}}\right)\right.\right. \\
\left.\left.+w(z-h, y) \frac{\sigma^{2}}{2 h^{2}}+\min \left(e^{z}, M\right) u\right)\right]-w(z, y)=0 .
\end{gathered}
$$

TheOREM 4.1. Let $w_{k, h}^{M}$ denote the solution to (12). Then, as $(h, k) \rightarrow 0$, the sequence $w_{k, h}^{M}$ converges locally uniformly on $\mathbb{R} \times[0, N]$ to the unique viscosity solution $w_{M}$ of (10).

REMARK 4.2. Using a change of variable argument, $v_{M}(x, y)=w_{M}(\log x, y)$ for $(x, y) \in(0,+\infty) \times[0, N]$ and that $\left(v_{M}\right)_{M}$ converges to $v$, the unique viscosity solution of (4). Finally,

$$
\lim _{M \rightarrow \infty} \lim _{h \rightarrow 0, k \rightarrow 0} w_{h, k}^{M}(\log x, y)=v(x, y) \text { for }(x, y) \in(0,+\infty) \times[0, N] .
$$


5. Numerical Demonstration. In this section we provide some numerical experiments to demonstrate the approximation of the value function and the optimal control policy for a stock $X(t)$. The data are specified as follows: The return rate $\mu=0.09$, volatility rate $\sigma=0.2$, and the discount rate $\rho=0.1$. As shown in Section 3 , the optimal selling strategy is given by (5). Our numerical experiments confirm such a finding. The value function and the optimal strategy are plotted in Figure 1.
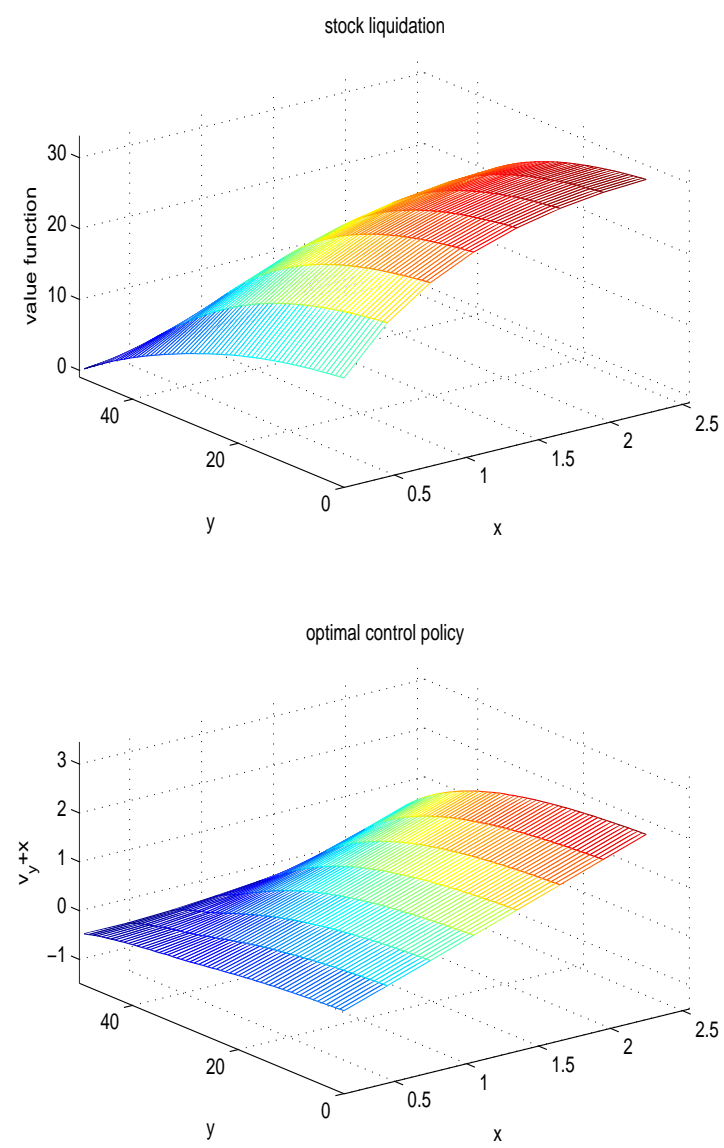

FIG. 1. Value function $v(x, y)$ and graph of the map $v_{y}+x$ that defines the optimal control policy

To gain further insight, we plot the different values of $x$ and $y$ and trace out the curve $\partial v(x, y) / \partial y+x$ in Figure 2. This curve separates the entire region into two parts, namely no sell region and sell region determined by (5). Note that in this paper, we formulated the problem as an optimal control problem with state constraints. It could also be formulated as an optimal stopping problem. Then naturally, one would expect to get a "free boundary." The curve $\partial v(x, y) / \partial y+x$ presents just this "free boundary." 
The threshold-type policy makes the optimal strategy appealing for market practice owing to its simple structure.

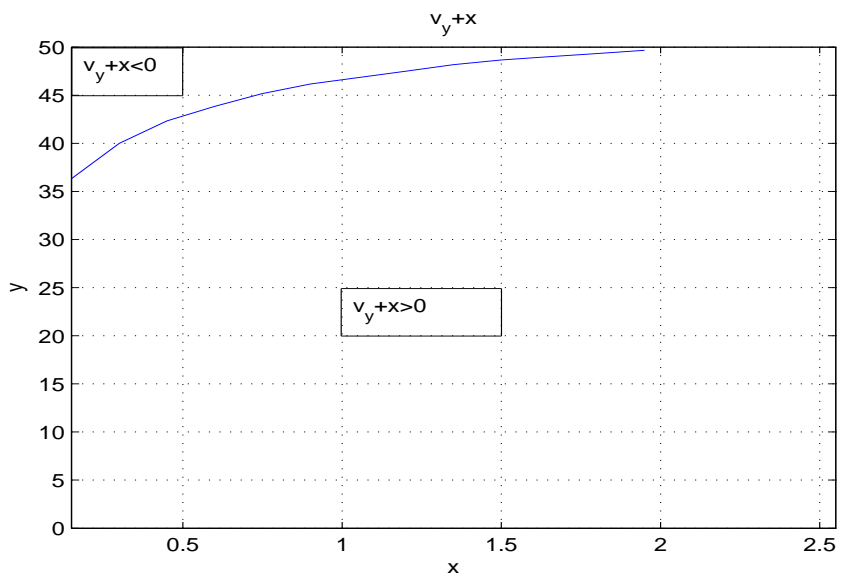

FIG. 2. graph of $v_{y}+x=0$.

In addition, we plotted the curves of $\partial v(x, y) / \partial y+x=0$ in Figure 3 with $\rho=0.2$, $\sigma=0.38$, and $\mu$ varying and taking values $0.3,0.4,0.5$, and 0.6 . This suggests the monotonicity of $u^{*}(x, y)$ with respect to $\mu$, i.e., the larger the $\mu$ the smaller the $u^{*}(x, y)$ representing less selling pressure with a larger return rate.

Similarly, in Figure 4, we plot the curves of $\partial v(x, y) / \partial y+x=0$ with $\mu=0.1$, $\sigma=0.4$, and $\rho=0.1,0.2,0.3,0.4$. The corresponding $u^{*}(x, y)$ is monotone increasing with respect to $\rho$. Namely, a larger $\rho$ results in deeper discount in the future and encourages more selling.

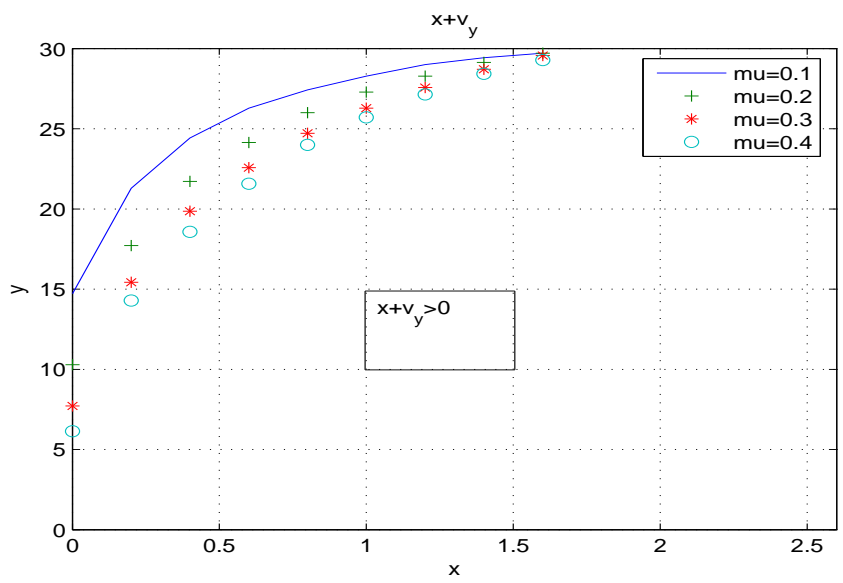

FIG. 3. Graph of $v_{y}+x=0$, with $\sigma=0.38, \rho=0.2$. 


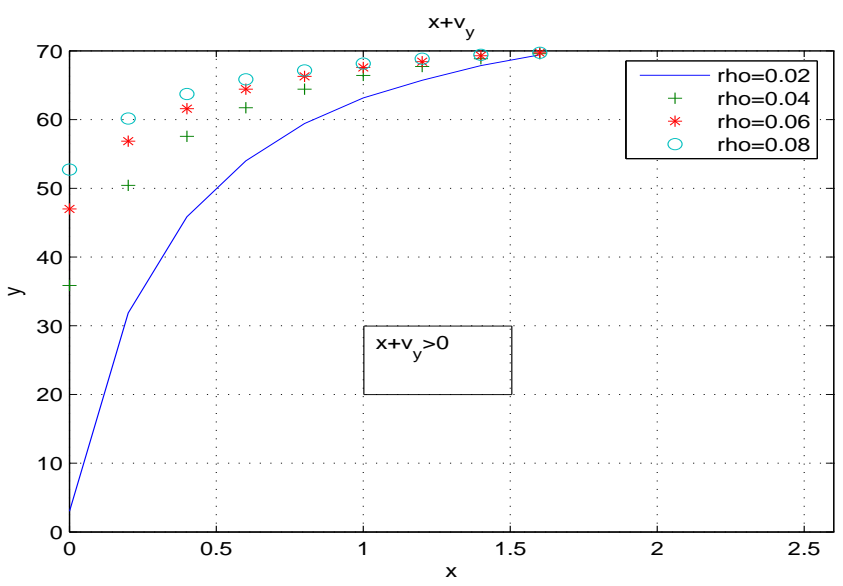

FIG. 4. Graph of $v_{y}+x=0$, with $\sigma=0.4, \mu=0.1$.

6. Conclusion. In this paper, we considered a selling strategy of a large block of stock under the framework of a fluid model and derived the optimal selling rate and a finite difference method for solving the associated HJB equation. It will be interesting to extend these results to incorporate taxes and transaction fees; see, for example, Cadenillas and Pliska [1] Constantinides [2], and Dammon and Spatt [3], for such a formulation and related references. Further thoughts and investigations are needed in this direction.

\section{Appendix A. Appendix: Proofs of Results.}

Proof of Lemma 3.1. To verify (a), note that for $0 \leq y_{1} \leq y_{2} \leq N, \mathcal{A}\left(x, y_{2}\right) \subset$ $\mathcal{A}\left(x, y_{1}\right)$. Given $u(\cdot) \in \mathcal{A}\left(x, y_{2}\right)$, then $u(\cdot) \in \mathcal{A}\left(x, y_{1}\right)$, and

$$
v\left(x, y_{1}\right) \geq J\left(x, y_{1}, u(\cdot)\right)=J\left(x, y_{2}, u(\cdot)\right)
$$

This implies $v\left(x, y_{1}\right) \geq v\left(x, y_{2}\right)$ because $u(\cdot)$ is arbitrary. Now we proceed to prove (b). Note that

$$
X(t)=x \exp \left(\left(\mu-\frac{\sigma^{2}}{2}\right) t+W(t)\right)
$$

Note also that for any $x_{1}>0$ and $x_{2}>0, \mathcal{A}\left(x_{1}, y\right)=\mathcal{A}\left(x_{2}, y\right)$, for $0 \leq y \leq N$. In addition, using Ito's rule, we can show $E X(t)=x e^{\mu t}$. Given $x_{1}>0$ and $x_{2}>0$, for 
each $u(\cdot) \in \mathcal{A}\left(x_{1}, y\right)=\mathcal{A}\left(x_{2}, y\right)$, we have

$$
\begin{aligned}
& \left|J\left(x_{1}, y, u(\cdot)\right)-J\left(x_{2}, y, u(\cdot)\right)\right| \\
& \quad=\left|x_{1}-x_{2}\right| E \int_{0}^{\infty} e^{-\rho t} \exp \left(\left(\mu-\frac{\sigma^{2}}{2}\right) t+W(t)\right) u(t) d t \\
& \quad \leq\left|x_{1}-x_{2}\right| E \int_{0}^{\infty} e^{-\rho t} \exp \left(\left(\mu-\frac{\sigma^{2}}{2}\right) t+W(t)\right) d t \\
& \quad=\left|x_{1}-x_{2}\right| \int_{0}^{\infty} e^{-(\rho-\mu) t} d t \\
& \quad=\frac{\left|x_{1}-x_{2}\right|}{\rho-\mu} .
\end{aligned}
$$

Since $u(\cdot)$ is arbitrary, we have

$$
\left|v\left(x_{1}, y\right)-v\left(x_{2}, y\right)\right| \leq \sup _{u(\cdot) \in \mathcal{A}\left(x_{1}, y\right)}\left|J\left(x_{1}, y, u(\cdot)\right)-J\left(x_{2}, y, u(\cdot)\right)\right| \leq \frac{\left|x_{1}-x_{2}\right|}{\rho-\mu} .
$$

It remains to show that $v(x, y)$ is Lipschitz in $y$. In view of (a), it suffices to show that for $0 \leq y_{1}<y_{2} \leq N$,

$$
v\left(x, y_{2}\right) \geq v\left(x, y_{1}\right)-(x+2)\left|y_{1}-y_{2}\right| .
$$

Let $u_{0}(\cdot) \in \mathcal{A}\left(x, y_{1}\right)$ such that $y_{1}+\int_{0}^{\infty} u_{0}(s) d s=N$ and

$$
v\left(x, y_{1}\right) \leq J\left(x, y_{1}, u_{0}(\cdot)\right)+\left|y_{1}-y_{2}\right| .
$$

Let $\tau=\inf \left\{t>0: \int_{0}^{t} u_{0}(s) d s=y_{2}-y_{1}\right\}$. Define

$$
\widetilde{u}(t)= \begin{cases}0 & \text { if } 0 \leq t<\tau, \\ u_{0}(t) & \text { if } t \geq \tau .\end{cases}
$$

Then, $\widetilde{u}(\cdot) \in \mathcal{A}\left(x, y_{2}\right)$. Moreover,

$$
\begin{aligned}
\left|J\left(x, y_{1}, u_{0}(\cdot)\right)-J\left(x, y_{2}, \widetilde{u}(\cdot)\right)\right| & \leq E \int_{0}^{\infty} e^{-\rho t} X(t)\left|u_{0}(t)-\widetilde{u}(t)\right| d t \\
& =E \int_{0}^{\tau} e^{-\rho t} X(t) u_{0}(t) d t .
\end{aligned}
$$

We next verify

$$
E \int_{0}^{\tau} e^{-\rho t} X(t) u_{0}(t) d t \leq(x+1)\left|y_{1}-y_{2}\right| .
$$

In fact, using integration by parts, we have

$$
\begin{aligned}
& E \int_{0}^{\tau} e^{-\rho t} X(t) u_{0}(t) d t \\
& =E\left[e^{-\rho \tau} X(\tau) \int_{0}^{\tau} u_{0}(s) d s\right]+(\rho-\mu) E \int_{0}^{\tau} e^{-\rho t}\left(\int_{0}^{\tau} u_{0}(s) d s\right) X(t) d t .
\end{aligned}
$$

Recall that $\rho>\mu$. It follows that

$$
E\left[e^{-\rho \tau} X(\tau) \int_{0}^{\tau} u_{0}(s) d s\right] \leq\left(y_{2}-y_{1}\right) E\left[e^{-\rho \tau} X(\tau)\right] \leq x\left|y_{1}-y_{2}\right| .
$$


Similarly, we have

$$
\begin{aligned}
E \int_{0}^{\tau} e^{-\rho t}\left(\int_{0}^{\tau} u_{0}(s) d s\right) X(t) d t & \leq\left(y_{2}-y_{1}\right) E \int_{0}^{\tau} e^{-\rho t} X(t) d t \\
& \leq\left(y_{2}-y_{1}\right) E \int_{0}^{\infty} e^{-\rho t} X(t) d t \\
& =\frac{\left|y_{1}-y_{2}\right|}{\rho-\mu}
\end{aligned}
$$

Therefore,

$$
\left|J\left(x, y_{1}, u_{0}(\cdot)\right)-J\left(x, y_{2}, \widetilde{u}(\cdot)\right)\right| \leq(x+1)\left|y_{1}-y_{2}\right| .
$$

It follows that

$$
\begin{aligned}
v\left(x, y_{2}\right) & \geq J\left(x, y_{2}, \widetilde{u}(\cdot)\right) \\
& \geq J\left(x, y_{1}, u_{0}(\cdot)\right)-(x+1)\left|y_{1}-y_{2}\right| \\
& \geq v\left(x, y_{1}\right)-(x+2)\left|y_{1}-y_{2}\right| .
\end{aligned}
$$

This completes the proof.

Proof of Theorem 3.2. For any stopping time $\tau$, the dynamic programming principe implies that

$$
v(x, y)=\max _{u(\cdot) \in \mathcal{A}} E\left\{\int_{0}^{\tau} e^{-\rho t} X(t) u(t) d t+e^{-\rho \tau} v(X(\tau), Y(\tau))\right\} .
$$

Given $(X(0), Y(0))=\left(x_{0}, y_{0}\right) \in(0, \infty) \times[0, N]$, let $\phi(x, y) \in C^{2}((0, \infty) \times[0, N])$, such that $v(x, y)-\phi(x, y)$ has a local minimum in a neighborhood $N\left(x_{0}, y_{0}\right)$ of $\left(x_{0}, y_{0}\right)$. Without loss of generality we can assume that $v\left(x_{0}, y_{0}\right)-\phi\left(x_{0}, y_{0}\right)=0$. Let $\tau_{0}$ be a stopping time such that for $0 \leq t \leq \tau_{0},(X(t), Y(t)) \in N\left(x_{0}, y_{0}\right)$. Consider the control $u(t)=u \in \Gamma$, for $t \in\left[0, \tau_{0}\right]$, where $u$ is a constant such that $u(\cdot) \in \mathcal{A}\left(x_{0}, y_{0}\right)$. Moreover, for $t \in\left[0, \tau_{0}\right], v(X(t), Y(t))-\phi(X(t), Y(t)) \geq 0$. Given $\theta>0$, using (13), we have

$$
v\left(x_{0}, y_{0}\right) \geq E \int_{0}^{\theta \wedge \tau_{0}} e^{-\rho s} X(s) u(s) d s+E e^{-\rho\left(\theta \wedge \tau_{0}\right)} v\left(X\left(\theta \wedge \tau_{0}\right), Y\left(\theta \wedge \tau_{0}\right)\right) .
$$

Using Dynkin's formula, we have

$$
\begin{aligned}
& E e^{-\rho\left(\theta \wedge \tau_{0}\right)} \phi\left(X\left(\theta \wedge \tau_{0}\right), Y\left(\theta \wedge \tau_{0}\right)\right)-\phi\left(x_{0}, y_{0}\right) \\
& =E \int_{0}^{\theta \wedge \tau_{0}} e^{-\rho s}\left\{\mu X(s) \frac{\partial \phi(X(s), Y(s))}{\partial x}+u(s) \frac{\partial \phi(X(s), Y(s))}{\partial y}\right. \\
& \left.\quad+\frac{1}{2} \sigma^{2} X^{2}(s) \frac{\partial^{2} \phi(X(s), Y(s))}{\partial x^{2}}-\rho \phi(X(s), Y(s))\right\} d s .
\end{aligned}
$$

It follows that

$$
\begin{aligned}
& E e^{-\rho\left(\theta \wedge \tau_{0}\right)} v\left(X\left(\theta \wedge \tau_{0}\right), Y\left(\theta \wedge \tau_{0}\right)\right)-v\left(x_{0}, y_{0}\right) \\
& \geq E \int_{0}^{\theta \wedge \tau_{0}} e^{-\rho s}\left\{\mu X(s) \frac{\partial \phi(X(s), Y(s))}{\partial x}+u(s) \frac{\partial \phi(X(s), Y(s))}{\partial y}\right. \\
& \left.\quad+\frac{1}{2} \sigma^{2} X^{2}(s) \frac{\partial^{2} \phi(X(s), Y(s))}{\partial x^{2}}-\rho v(X(s), Y(s))\right\} d s .
\end{aligned}
$$


Combining (14) and (15), we obtain

$$
\begin{aligned}
0 \geq & E \int_{0}^{\theta \wedge \tau_{0}} e^{-\rho s}\left\{\mu X(s) \frac{\partial \phi(X(s), Y(s))}{\partial x}+u(s) \frac{\partial \phi(X(s), Y(s))}{\partial y}\right. \\
& \left.+\frac{1}{2} \sigma^{2} X^{2}(s) \frac{\partial^{2} \phi(X(s), Y(s))}{\partial x^{2}}+u(s) X(s)-\rho v(X(s), Y(s))\right\} d s .
\end{aligned}
$$

Letting $\theta \rightarrow 0$ allows us to conclude that

$$
\begin{array}{r}
\max _{u \in \Gamma}\left[\mu x_{0} \frac{\partial \phi\left(x_{0}, y_{0}\right)}{\partial x}+u \frac{\partial \phi\left(x_{0}, y_{0}\right)}{\partial y}+\frac{1}{2} \sigma^{2} x_{0}^{2} \frac{\partial^{2} \phi\left(x_{0}, y_{0}\right)}{\partial x^{2}}+u x_{0}\right] \\
-\rho v\left(x_{0}, y_{0}\right) \leq 0 .
\end{array}
$$

Thus, $v$ is a viscosity supersolution. Next, we show that $v$ is viscosity subsolution on $(0, \infty) \times(0, N)$. For suppose not, $v$ is not a viscosity subsolution. Then there exists $\left(x_{0}, y_{0}\right) \in(0, \infty) \times(0, N)$ and $\delta>0$ such that for all control $u(\cdot) \in \mathcal{A}$,

$$
\mu x \frac{\partial \psi(x, y)}{\partial x}+u \frac{\partial \psi(x, y)}{\partial y}+\frac{1}{2} \sigma^{2} x^{2} \frac{\partial^{2} \psi(x, y)}{\partial x^{2}}+u x-\rho v(x, y) \leq-\delta
$$

in the neighborhood $N\left(x_{0}, y_{0}\right)$, where $\psi(x, y) \in C^{2}((0, \infty) \times(0, N))$, such that $v(x, y)-$ $\psi(x, y)$ attains its maximum at $\left(x_{0}, y_{0}\right)$ in $N\left(x_{0}, y_{0}\right)$. Without loss of generality we can assume that $v\left(x_{0}, y_{0}\right)-\psi\left(x_{0}, y_{0}\right)=0$. Let $u(\cdot) \in \mathcal{A}\left(x_{0}, y_{0}\right)$. Let $\tau$ be a stopping time such that for $0 \leq s \leq \tau,(X(s), Y(s)) \in N\left(x_{0}, y_{0}\right), v(X(s), Y(s))-\psi(X(s), Y(s)) \leq 0$. We have

$$
\begin{aligned}
J\left(x_{0}, y_{0}, u(\cdot)\right) & \leq E \int_{0}^{\tau} e^{-\rho s} X(s) u(s) d s+E e^{-\rho \tau} v(X(\tau), Y(\tau)) \\
& \leq E \int_{0}^{\tau} e^{-\rho s} X(s) u(s) d s+E e^{-\rho \tau} \psi(X(\tau), Y(\tau)) .
\end{aligned}
$$

Using (18), we have

$$
\begin{aligned}
J\left(x_{0}, y_{0}, u(\cdot)\right) \leq & E \int_{0}^{\tau} e^{-\rho s}\left(-\delta+\rho v(X(s), Y(s))-\mu X(s) \frac{\partial \psi(X(s), Y(s))}{\partial x}\right. \\
& \left.-u \frac{\partial \psi(X(s), Y(s))}{\partial y}-\frac{1}{2} \sigma^{2} X(s)^{2} \frac{\partial^{2} \psi(X(s), Y(s))}{\partial x^{2}}\right) d s \\
& +E e^{-\rho \tau} \psi(X(\tau), Y(\tau)) \\
\leq & E \int_{0}^{\tau} e^{-\rho s}\left(-\delta+\rho \psi(X(s), Y(s))-\mu X(s) \frac{\partial \psi(X(s), Y(s))}{\partial x}\right. \\
& \left.-u \frac{\partial \psi(X(s), Y(s))}{\partial y}-\frac{1}{2} \sigma^{2} X^{2}(s) \frac{\partial^{2} \psi(X(s), Y(s))}{\partial x^{2}}\right) d s \\
& +E e^{-\rho \tau} \psi(X(\tau), Y(\tau)) \\
\leq & -E \int_{0}^{\tau} e^{-\rho s} \delta d s+\psi\left(x_{0}, y_{0}\right) \\
= & -E \int_{0}^{\tau} e^{-\rho s} \delta d s+v\left(x_{0}, y_{0}\right) .
\end{aligned}
$$


Taking the supremum over all admissible control $u \in \mathcal{A}$ we have

$$
v\left(x_{0}, y_{0}\right) \leq-E \int_{0}^{\tau} e^{-\rho s} \delta d s+v\left(x_{0}, y_{0}\right) .
$$

This contradicts to $\delta>0$. Therefore $v(x, y)$ is a viscosity subsolution. Finally, the uniqueness can be obtained using the ideas of Soner [13] and Ishii [7].

Convergence of the Finite Difference Method. Define a mapping $S_{M}$ : $(0,1) \times(0,1) \times \mathbb{R} \times[0, N] \times \mathbb{R} \times B(\mathbb{R} \times[0, N]) \rightarrow \mathbb{R}$

$$
\begin{aligned}
S_{M}(k, h, z, y, t, w)= & \max _{u \in \Gamma}\left[h\left(\rho+\left(\mu-\frac{\sigma^{2}}{2}\right) \frac{1}{h}+\frac{\sigma^{2}}{h^{2}}+\frac{u}{k}\right) t\right. \\
& -h\left(w(z, y+k) \frac{u}{k}+w(z+h, y)\left(\left(\mu-\frac{\sigma^{2}}{2}\right) \frac{1}{h}+\frac{\sigma^{2}}{2 h^{2}}\right)\right. \\
& \left.\left.+w(z-h, y) \frac{\sigma^{2}}{2 h^{2}}+\min \left(e^{z}, M\right) u\right)\right] .
\end{aligned}
$$

Then, (11) is equivalent to $S_{M}(k, h, z, y, w(z, y), w)=0$. Moreover, note that the coefficient of $w$ in $S_{M}$ is negative. This implies that $S_{M}$ is monotone, i.e., for all $t \in \mathbb{R}, k, h \in(0,1), z \in \mathbb{R}, y \in[0, N]$, and $w_{1}, w_{2} \in B(\mathbb{R} \times[0, N])$

$$
S_{M}\left(k, h, z, y, t, w_{1}\right) \leq S_{M}\left(k, h, z, y, t, w_{2}\right) \text { whenever } w_{2} \leq w_{1} .
$$

Definition A.1. The scheme $S_{M}$ is said to be consistent if, for every $z \in \mathbb{R}$, $y \in[0, N]$, and for every test function $\eta(\cdot, \cdot) \in \mathcal{C}^{1,2}(\mathbb{R} \times[0, N])$,

$$
\lim _{\xi \rightarrow z, \zeta \rightarrow y k \downarrow 0, \varepsilon \rightarrow 0, h \downarrow 0} \frac{S_{M}(k, h, \xi, \zeta, \eta(\xi, \zeta)+\varepsilon, \eta+\varepsilon)}{h}=\mathcal{H}_{M}\left(z, y, \eta, \mathrm{D}_{z} \eta, \mathrm{D}_{y} \eta, \mathrm{D}_{z}^{2} \eta\right) .
$$

Lemma A.2. The scheme $S_{M}$ is consistent.

Proof. Let $\eta(\cdot, \cdot) \in \mathcal{C}^{1,2}(\times \mathbb{R} \times[0, N])$. We write

$$
\begin{aligned}
\frac{S_{M}(k, h, \xi, \zeta, \eta(\xi, \zeta), \eta)}{h}= & \max _{u \in \Gamma}\left[\left(\rho+\left(\mu-\frac{\sigma^{2}}{2}\right) \frac{1}{h}+\frac{\sigma^{2}}{h^{2}}+\frac{u}{k}\right) \eta(\xi, \zeta)\right. \\
- & \left(\eta(\xi, \zeta+k) \frac{u}{k}+\eta(\xi+h, \zeta)\left(\left(\mu-\frac{\sigma^{2}}{2}\right) \frac{1}{h}\right.\right. \\
& \left.\left.\left.+\frac{\sigma^{2}}{2 h^{2}}\right)+\eta(\xi-h, \zeta) \frac{\sigma^{2}}{2 h^{2}}+\min \left(e^{\xi}, M\right) u\right)\right] .
\end{aligned}
$$

Sending $\xi \rightarrow z, \zeta \rightarrow y, k \rightarrow 0, \varepsilon \rightarrow 0$, and $h \rightarrow 0$, we can show that

$$
\frac{S_{M}(k, h, \xi, \zeta, \eta(\xi, \zeta)+\varepsilon, \eta+\varepsilon)}{h} \rightarrow \mathcal{H}_{M}\left(z, y, \eta, \mathrm{D}_{z} \eta, \mathrm{D}_{y} \eta, \mathrm{D}_{z}^{2} \eta\right) .
$$

This completes the proof.

Note that the equation $S_{M}(k, h, z, y, w(z, y), v)=0$ is equivalent to the equation

$$
\begin{aligned}
w(z, y)= & \max _{u \in \Gamma}\left[\frac { 1 } { \rho + ( \mu - \frac { \sigma ^ { 2 } } { 2 } ) \frac { 1 } { h } + \frac { \sigma ^ { 2 } } { h ^ { 2 } } + \frac { u } { k } } \left(w(z, y+k) \frac{u}{k}\right.\right. \\
& +w(z+h, y)\left(\left(\mu-\frac{\sigma^{2}}{2}\right) \frac{1}{h}+\frac{\sigma^{2}}{2 h^{2}}\right)+w(z-h, y) \frac{\sigma^{2}}{2 h^{2}} \\
& \left.\left.+\min \left(e^{z}, M\right) u\right)\right] .
\end{aligned}
$$


We define an operator $\mathcal{T}_{h, k}$ on $B(\mathbb{R} \times[0, N])$ as follows,

$$
\begin{aligned}
\mathcal{T}_{h, k} w(z, y)= & \max _{u \in \Gamma}\left[\frac { 1 } { \rho + ( \mu - \frac { \sigma ^ { 2 } } { 2 } ) \frac { 1 } { h } + \frac { \sigma ^ { 2 } } { h ^ { 2 } } + \frac { u } { k } } \left(w(z, y+k) \frac{u}{k}\right.\right. \\
& +w(z+h, y)\left(\left(\mu-\frac{\sigma^{2}}{2}\right) \frac{1}{h}+\frac{\sigma^{2}}{2 h^{2}}\right)+w(z-h, y) \frac{\sigma^{2}}{2 h^{2}} \\
& \left.\left.+\min \left(e^{z}, M\right) u\right)\right] .
\end{aligned}
$$

Lemma A.3. For each $k$ and $h, \mathcal{T}_{k, h}$ is a contraction map.

Proof. To prove $\mathcal{T}_{k, h}$ is a contraction, we need to show that there exists $0<\beta<1$ such that

$$
\left\|\mathcal{T}_{k, h} f-\mathcal{T}_{k, h} g\right\| \leq \beta\|f-g\| \text { for all } f, g \in B(\mathbb{R} \times[0, N]),
$$

where $\|\cdot\|$ is the sup norm. Let us define $c_{h, k}$ by

$$
c_{h, k}(u)=\rho+\left(\mu-\frac{\sigma^{2}}{2}\right) \frac{1}{h}+\frac{\sigma^{2}}{h^{2}}+\frac{u}{k} .
$$

Note that

$$
\begin{aligned}
\mathcal{T}_{k, h} \phi & (z, y)-\mathcal{T}_{k, h} \psi(z, y) \\
= & \max _{u \in \Gamma}\left[\frac { 1 } { c _ { h , k } ( u ) } \left(\phi(z, y+k) \frac{u}{k}+\phi(z+h, y)\left(\left(\mu-\frac{\sigma^{2}}{2}\right) \frac{1}{h}+\frac{\sigma^{2}}{2 h^{2}}\right)\right.\right. \\
& \left.\left.+\phi(z-h, y) \frac{\sigma^{2}}{2 h^{2}}+\min \left(e^{z}, M\right) u\right)\right] \\
& \quad-\max _{u \in \Gamma}\left[\frac { 1 } { c _ { h , k } ( u ) } \left(\psi(z, y+k) \frac{u}{k}+\psi(z+h, y)\left(\left(\mu-\frac{\sigma^{2}}{2}\right) \frac{1}{h}+\frac{\sigma^{2}}{2 h^{2}}\right)\right.\right. \\
& \left.\left.+\psi(z-h, y) \frac{\sigma^{2}}{2 h^{2}}+\min \left(e^{z}, M\right) u\right)\right] .
\end{aligned}
$$

This implies that for all $z, y$,

$$
\begin{aligned}
& \left|\mathcal{T}_{k, h} \phi(z, y)-\mathcal{T}_{k, h} \psi(z, y)\right| \\
& \quad \leq \max _{u \in \Gamma}\left[\frac{\frac{u}{k}+\left(\mu-\frac{\sigma^{2}}{2}\right) \frac{1}{h}+\frac{\sigma^{2}}{h^{2}}}{c_{h, k}(u)}\right]\|\phi-\psi\|
\end{aligned}
$$

In addition, note that

$$
\frac{\frac{u}{k}+\left(\mu-\frac{\sigma^{2}}{2}\right) \frac{1}{h}+\frac{\sigma^{2}}{h^{2}}}{c_{h, k}(u)}=\frac{\frac{u}{k}+\left(\mu-\frac{\sigma^{2}}{2}\right) \frac{1}{h}+\frac{\sigma^{2}}{h^{2}}}{\rho+\left(\mu-\frac{\sigma^{2}}{2}\right) \frac{1}{h}+\frac{\sigma^{2}}{h^{2}}+\frac{u}{k}}<1 .
$$

Since $u$ takes values in $[0,1]$,

$$
\max _{u \in \Gamma} \frac{\frac{u}{k}+\left(\mu-\frac{\sigma^{2}}{2}\right) \frac{1}{h}+\frac{\sigma^{2}}{h^{2}}}{c_{h, k}(u)}<1 .
$$

Therefore,

$$
\left\|\mathcal{T}_{k, h} \phi-\mathcal{T}_{k, h} \psi\right\|<\beta_{h, k}\|\phi-\psi\|,
$$


with

$$
\beta=\beta_{h, k}=\max _{u \in \Gamma} \frac{\frac{u}{k}+\left(\mu-\frac{\sigma^{2}}{2}\right) \frac{1}{h}+\frac{\sigma^{2}}{h^{2}}}{c_{h, k}(u)}=\max _{u \in \Gamma} \frac{c_{h, k}(u)-\rho}{c_{h, k}(u)}<1 .
$$

Definition A.4. The scheme $S_{M}$ is said to be stable if for every $h, k \in(0,1)$, there exists a bounded solution $w_{h, k} \in B(\mathbb{R} \times[0, N])$ to the equation

$$
S_{M}(k, h, z, y, w(z, y), w)=0,
$$

with the bound independent of $k$, and $h$.

REMARK A.5. By the Banach fixed point theorem, the strict contraction $\mathcal{T}_{h k}$ has a unique fixed point that we denote by $w_{h, k}^{M}$. Given any function $w_{0} \in B(\mathbb{R} \times[0, N])$, we construct a sequence as follows, $w_{n+1}=\mathcal{T}_{h k} w_{n}$ for $n \geq 0$. It is clear that

$$
\lim _{n \rightarrow \infty} w_{n}=w_{h, k}^{M}
$$

Moreover, note that

$$
\begin{aligned}
w_{n+1}(z, y)= & \max _{u \in \Gamma}\left[\frac { 1 } { c _ { h , k } ( u ) } \left(w_{n}(z, y+k) \frac{u}{k}\right.\right. \\
& +w_{n}(z+h, y)\left(\left(\mu-\frac{\sigma^{2}}{2}\right) \frac{1}{h}+\frac{\sigma^{2}}{2 h^{2}}\right) \\
& \left.\left.\quad+w_{n}(z-h, y) \frac{\sigma^{2}}{2 h^{2}}+\min \left(e^{z}, M\right) u\right)\right] \\
\leq & \beta_{h, k}\left\|w_{n}\right\|+\frac{1}{c_{h, k}(1)} M .
\end{aligned}
$$

In addition, we have

$$
\beta_{h, k}=\max _{u \in \Gamma} \frac{c_{h, k}(u)-\rho}{c_{h, k}(u)} \leq \max _{u \in \Gamma} \frac{c_{h, k}(u)-\rho}{c_{h, k}(u)}=\frac{c_{h, k}(1)-\rho}{c_{h, k}(1)}<1
$$

because $c_{h, k}(u)$ is an affine function of $u$ and $\Gamma=[0,1]$. This implies that

$$
\left\|w_{n+1}\right\| \leq \frac{c_{h, k}(1)-\rho}{c_{h, k}(1)}\left\|w_{n}\right\|+\frac{1}{c_{h, k}(1)} M .
$$

From (26), we deduce that

$$
\left\|w_{n+1}\right\| \leq\left(\frac{c_{h, k}(1)-\rho}{c_{h, k}(1)}\right)^{n+1}\left\|w_{0}\right\|+\frac{M}{c_{h, k}(1)} \sum_{i=0}^{n}\left(\frac{c_{h, k}(1)-\rho}{c_{h, k}(1)}\right)^{i} .
$$

Taking the limit as $n \rightarrow \infty$, we obtain

$$
\left\|w_{h, k}^{M}\right\| \leq \frac{M}{c_{h, k}(1)} \frac{1}{1-\frac{c_{h, k}(1)-\rho}{c_{h, k}(1)}}=\frac{M}{\rho} .
$$

This implies the stability of the scheme $S_{M}$. 
Proof of Theorem 4.1. Define

$$
\begin{aligned}
w_{M}^{*}(x, y) & =\limsup _{\xi \rightarrow x, \zeta \rightarrow y, k \downarrow 0, h \downarrow 0} w_{k, h}^{M}(\xi, \zeta) \text { and } \\
w_{* M}(x, y) & =\liminf _{\xi \rightarrow x, \zeta \rightarrow y, k \downarrow 0, h \downarrow 0} w_{k, h}^{M}(\xi, \zeta) .
\end{aligned}
$$

We claim that $w_{M}^{*}$ and $w_{* M}$ are sub- and supersolutions of (10), respectively.

Since the argument for that of $w_{* M}$ is similar, to prove this claim, we only consider $w_{M}^{*}$. We want to show:

$$
\mathcal{H}_{M}\left(x_{0}, y_{0}, w_{M}^{*}\left(x_{0}, y_{0}\right), \mathrm{D}_{x} \Phi\left(x_{0}, y_{0}\right), \mathrm{D}_{y} \Phi\left(x_{0}, y_{0}\right), \mathrm{D}_{x}^{2} \Phi\left(x_{0}, y_{0}\right)\right) \leq 0
$$

for any test function $\Phi \in \mathcal{C}^{1,2}(\mathbb{R} \times[0, N])$ such that $\left(x_{0}, y_{0}\right)$ is a strictly local maximum of $w_{M}^{*}(x, y)-\Phi(x, y)$. Without loss of generality, we may assume that $w_{M}^{*}\left(x_{0}, y_{0}\right)=$ $\Phi\left(x_{0}, y_{0}\right)$ and because of the stability of our scheme we can also assume that $\Phi \geq$ $2 \sup _{k, h}\left\|w_{k, h}^{M}\right\|$ outside of the ball $B\left(\left(x_{0}, y_{0}\right), r\right)$ where $r>0$ is such that

$$
w_{M}^{*}(x, y)-\Phi(t, x) \leq 0=w_{M}^{*}\left(x_{0}, y_{0}\right)-\Phi\left(t_{0}, x_{0}\right) \text { in } B\left(\left(x_{0}, y_{0}\right), r\right) .
$$

This implies that there exist sequences $k_{n}>0, h_{n}>0$, and $\left(\xi_{n}, \zeta_{n}\right) \in \mathbb{R} \times[0, N]$ such that as $n \rightarrow \infty$, we have

$$
\begin{aligned}
& k_{n} \rightarrow 0, \quad h_{n} \rightarrow 0, \quad \xi_{n} \rightarrow x_{0}, \quad \zeta_{n} \rightarrow y_{0}, w_{k_{n}, h_{n}}^{M}\left(\xi_{n}, \zeta_{n},\right) \rightarrow w_{M}^{*}\left(x_{0}, y_{0}\right), \\
& \text { and }\left(\xi_{n}, \zeta_{n}\right) \text { is a global maximum } w_{k_{n}, h_{n}}^{M}-\Phi
\end{aligned}
$$

Denote $\varepsilon_{n}=w_{k_{n}, h_{n}}^{M}\left(\xi_{n}, \zeta_{n}\right)-\Phi\left(\xi_{n}, \zeta_{n}\right)$. Clearly $\varepsilon_{n} \rightarrow 0$ and

$$
w_{k_{n}, h_{n}}^{M}(x, y) \leq \Phi(x, y)+\varepsilon_{n} \text { for all }(x, y) \in \mathbb{R} \times[0, N] .
$$

We know that

$$
S_{M}\left(k_{n}, h_{n}, \xi_{n}, \zeta_{n}, w_{k_{n}, h_{n}}^{M}\left(\xi_{n}, \zeta_{n}\right), w_{k_{n}, h_{n}}^{M}\right)=0 .
$$

The monotonicity of $S_{M}$ and (29) imply

$$
\begin{aligned}
& S_{M}\left(k_{n}, h_{n}, \xi_{n}, \zeta_{n}, \Phi\left(\xi_{n}, \zeta_{n}\right)+\varepsilon_{n}, \Phi+\varepsilon_{n}\right) \\
& \quad \leq S_{M}\left(k_{n}, h_{n}, \xi_{n}, \zeta_{n}, w_{k_{n}, h_{n}}^{M}\left(\xi_{n}, \zeta_{n}\right), w_{k_{n}, h_{n}}^{N}\right)=0 .
\end{aligned}
$$

Therefore,

$$
\lim _{n} \frac{S_{M}\left(k_{n}, h_{n}, \xi_{n}, \zeta_{n}, \Phi\left(\xi_{n}, \zeta_{n}\right)+\varepsilon_{n}, \Phi+\varepsilon_{n}\right)}{h_{n}} \leq 0
$$

so

$$
\begin{aligned}
& \mathcal{H}_{M}\left(x_{0}, y_{0}, w_{M}^{*}\left(x_{0}, y_{0}\right), \mathrm{D}_{x} \Phi\left(x_{0}, y_{0}\right), \mathrm{D}_{y} \Phi\left(x_{0}, y_{0}\right), \mathrm{D}_{x}^{2} \Phi\left(x_{0}, y_{0}\right)\right) \\
& =\lim _{\xi \rightarrow x_{0}, \zeta_{n} \rightarrow y_{0}, k_{n} \downarrow 0, \varepsilon \downarrow 0, h \downarrow 0} \frac{S_{M}\left(k_{n}, h_{n}, \xi_{n}, \zeta_{n}, \Phi\left(\xi_{n}, \zeta_{n}\right)+\varepsilon_{n}, \Phi+\varepsilon_{n}\right)}{h_{n}} \leq 0 .
\end{aligned}
$$


This proves that $w_{M}^{*}$ is a viscosity subsolution and, similarly we can prove that $w_{* M}$ is a viscosity supersolution. Thus, using the uniqueness of the viscosity solution, we see that $w_{M}=w_{M}^{*}=w_{* M}$. Therefore, we conclude that the sequence $\left(w_{h, k}^{M}\right)_{h, k}$ converges locally uniformly to $w_{M}$ as desired.

\section{REFERENCES}

[1] A. Cadenillas and S. R. Pliska, Optimal trading of a security when there are taxes and transaction costs, Finance \& Stochastics, 3(1999), pp. 137-165.

[2] G. M. Constantinides, Capital market equilibrium with personal tax, Econometrica, 51(1983), pp. 611-636.

[3] R. M. Dammon And C. S. Spatt, The optimal trading and pricing of securities with asymetric capital gains taxes and transaction costs, Rev. Financial Studies, 9(1996), pp. 921-952.

[4] W. H. Fleming and R. Rishel, Deterministic and Stochastic Optimal Control, SpringerVerlag, New York, 1975.

[5] X. Guo And Q. Zhang, Optimal selling rules in a regime switching model, IEEE Trans. on Automatic Control, 50(2005), pp. 1450-1455.

[6] K. Helmes, Computing optimal selling rules for stocks using linear programming, Mathematics of Finance, pp. 87-198. G. Yin and Q. Zhang (Eds), American Mathematical Society, 2004.

[7] H. IsHII, Uniqueness of unbounded viscosity solutions of Hamilton-Jacobi equations, Indiana Univ. Math. J., 33(1984), pp. 721-748.

[8] H. P. McKean, A free boundary problem for the heat equation arising from a problem in mathematical economics, Indust. Management Rev., 60(1960), pp. 32-39.

[9] B. ØKsendal, Stochastic Differential Equations, Springer, New York, 1998.

[10] M. PemY, Regime Switching Market Models and Applications, Ph.D. Thesis, University of Georgia, USA, 2005.

[11] M. Pemy And Q. Zhang, Optimal stock liquidation in a regime switching model with finite time horizon, J. Math. Anal. Appl., 321(2006), pp. 537-552.

[12] D. D. YaO, Q. Zhang, X. Y. Zhou, A Regime-Switching Model for European Options, Stochastic Processes, Optimization, and Control Theory Applications in Financial Engineearing, Queueing Networks, and Manufacturing Systems, H. Yan, G. Yin and Q. Zhang (Eds.), in International Series in Operations Research and Management Sciences, Springer-SBM, New York, 2006.

[13] H. M. Soner, Optimal control with state space constraints II, SIAM J. Control Optim., 24(1986), pp. 1110-1122.

[14] G. Yin, R. H. LiU, AND Q. Zhang, Recursive algorithms for stock Liquidation: A stochastic optimization approach, SIAM J. Optim., 13(2002), pp. 240-263.

[15] G. Yin, Q. Zhang, F. Liu, R. H. Liu, And Y. Cheng, Stock liquidation via stochastic approximation using NASDAQ daily and intra-day data, Mathematical Finance, 16(2006), pp. 217-236.

[16] Q. Zhang, Stock trading: An optimal selling rules, SIAM J. Control Optim., 40(2001), pp.4-87.

[17] Q. Zhang, G. Yin, AND R. H. LiU, A near-optimal selling rule for a two-time-scale market model, Multiscale Modeling and Simulation: A SIAM Interdisciplinary J., 4(2005), pp. 172-193.

[18] X. Y. ZноU, Verification theorems within the framework of viscosity solutions, J. Math. Anal. Appl., 177(1993), pp. 208-225. 
